\title{
Solvent extraction of scandium from chlorination system with EHPAEH
}

\author{
Lan Jiang, Hongliang Yang*, and Yang Sun \\ Northeastern University(School of materials and metallurgy Northeastern University)
}

\begin{abstract}
The extraction of scandium from hydrochloric acid media with EHPAEH is investigated. Fe3+ in synthesized solution would reduce the purity of scandium, therefore, N235 is selected as extractant for separating $\mathrm{Fe} 3+$ and $\mathrm{Sc} 3+$. Experimental results indicated that separation factor $\beta \mathrm{Fe} / \mathrm{Sc}$ reached 5416.4 with the organic system consisting of $20 \mathrm{vol} \%$ trinoctylamine( $\mathrm{N} 235)$, phase volume ratio(O/A) of 1:1, extraction stage of 3 . Then mono(2-ethyl hexyl)-2-ethyl hexyl phosphonate(EHPAEH) is employed to extract $\mathrm{Sc} 3+$ from the leaching solution of $\mathrm{Fe} 3+$ free. With the organic phase containing $5 \mathrm{vol} \%$ EHPAEH, phase volume ratio O/A of 1:5, at $\mathrm{pH} 0.3$, nearly $99 \%$ scandium is extracted. Finally, the scandium-loaded organic phase is stripped with $4.5 \mathrm{~N}$ hydrochloric acid, under phase volume ratio of $1: 1$ at $65^{\circ} \mathrm{C}$.
\end{abstract}

\section{Introduction}

Scandium, an important Rare Earth element, is widely used in advanced ceramics, electric lighting source, electronic and other high-tech fields due to its excellent physical and chemical properties. In nature, independent scandium deposits are rare, it is usually associated with other metals and mainly recovered as by-product from residues, tailings and waste liquors in the smelting process of some metals such as rare earths, uranium, titanium, tungsten, aluminium, tantalum and niobium[1,2]. In this experiment, raw material is the residue originated in $\mathrm{TiCl}_{4}$ production by boiling chlorinating method (and the scandium compounds are enriched in it)[3].

Scandium in chlorination residue can be leached with hydrochloric acid. The methods of recovering scandium from synthesized solutions usually include solvent extraction[4-8] and ion exchange[4,9-11].

Solvent extraction with the characteristics of efficiency and economica is widely used in the enrichment and purification of many metals. Plenty of extractants can be used for scandium concentrate and separate it from other metal ions [2]. Acidic organophosphorus and neutral organophosphorus are two common types of extractants. Nearly $100 \%$ scandium is extracted from chloride solutions with $20 \mathrm{vol} \%$ di-2-ethylhexyl phosphoric acid(D2EHPA, in China used to call $\mathrm{P}_{204}$ )[5].

In this paper, $\mathrm{Fe}^{3+}$ is removed by $\mathrm{N}_{235}$ before extracting $\mathrm{Sc}^{3+}$ with EHPAEH. Experimental parameters which affecting extraction process, such as concentration of

*Corresponding author: 421589185@qq.com 
extractants, phase volume ratio $(\mathrm{O} / \mathrm{A})$, temperature and extraction stages are investigated in detail and the optimal experimental conditions are carried out.

\section{Experimental}

\subsection{Aqueous solution}

Synthetic leaching solution for this experiment was prepared by dissolving chlorination dust with $1 \mathrm{~mol} / \mathrm{L} \mathrm{HCl}$, liquid to solid ratio $(\mathrm{L} / \mathrm{S})$ of $2: 1$, at $75^{\circ} \mathrm{C}$ holding for $3 \mathrm{~h}$. (The concentration of $\mathrm{Sc}^{3+}$ and $\mathrm{Fe}^{3+}$ were respectively $238.3 \mathrm{mg} / \mathrm{L}$ and $3.1 \mathrm{~g} / \mathrm{L}$ ). All aqueous solutions were made up using distilled water.

\subsection{Organic reagents}

Extractant Trinoctylamine (N235) and Mono(2-ethyl hexyl)-2-ethyl hexyl phosphonate (EHPAEH) were of industrial grade. Modifier Sec-Octyl alcohol analytical grade was of analytical grade. Diluent sulfonated kerosene was of industrial grade. All reagents were directly used without further purification.

\subsection{Test produces}

Extraction and stripping experiments were carried out in a $125 \mathrm{ml}$ separating funnel by contacting the organic and aqueous phases under the given experimental condition including certain $\mathrm{pH}$, composition of organic phase, phase volume ratio O/A and temperature. The funnel was immersed in a temperature controlled water bath to keep temperature constant (and ensure a well mixed model between organic and aqueous solution). When the reaction reached equilibrium, organic phase and aqueous phase was separated, then ion concentrations in aqueous solution was analyzed.

\subsection{Test method and calculation formula}

Each test, about $10 \mathrm{~mL}$ aqueous solution was taken for chemical analysis. Inductively Coupled Plasma and Atomic Emission Spectroscopy (ICP-AES) was used to test the concentration of scandium. The model of $722 \mathrm{~s}$ spectrophotometer was used to test the concentration of $\mathrm{Fe}^{3+}$, and sulfosalicylic acid was served as chromogenic agent. Sartorius PHS-3 $\mathrm{pH}$ meter was employed to measure $\mathrm{pH}$ value in aqueous solution.

\section{Results and discussion}

\subsection{Removal of $\mathrm{Fe}^{3+}$ by Trinoctylamine $\left(\mathrm{N}_{235}\right)$}

The main impurities in the synthetic leaching solutions are iron, aluminum, manganese, calcium, titanium, manganese, etc. Preliminary experiments have shown that the co-extraction rate of $\mathrm{Mn}^{2+}$ and $\mathrm{Fe}^{3+}$ were relatively high when extracted with acidic organophosphorus. It will pose interference to the purity of scandium. $\mathrm{Mn}^{2+}$ can be removed by hydrolysis precipitation method due to the big difference of precipitation $\mathrm{pH}$. In this experiment, the effect of $\mathrm{N}_{235}$ concentration, modifier concentration, extraction stage on and separation factor were studied. 


\subsubsection{The effect of $N_{235}$ concentration}

Different concentrations of $\mathrm{N}_{235}$ in sulfonated kerosene between $5 \mathrm{vol} \%$ and $40 \mathrm{vol} \%$ were employed in the preliminary experiment and kept phase volume ratio $\mathrm{O} / \mathrm{A}$ of $1: 1$. The results shows that with the increase concentration of $\mathrm{N}_{235}$, the extraction rate of $\mathrm{Fe}^{3+}$ gradually increased, while it presents very little effect on the $\mathrm{Sc}^{3+}$ with the extraction rate always less than 2 vol\%. However, organic phase turns into a turbidity, when concentration exceeded $20 \mathrm{vol} \%$. The extraction rate only increased by $3.31 \%$ with $\mathrm{N}_{235}$ concentration increasing from $20 \mathrm{vol} \%$ to $30 \mathrm{vol} \%$ and the change of extraction rate and separation factor are very small after over $40 \mathrm{vol} \%$. The appropriate concentration of $\mathrm{N}_{235}$ in sulfonated kerosene is $20 \mathrm{vol} \%$ and it was set up in the following experiments.

Table 1. Effect of $\mathrm{N}_{235}$ concentration on extraction.

\begin{tabular}{|c|c|c|c|c|}
\hline No. & $\begin{array}{c}\mathrm{N}_{235} \\
\text { concentrations } \\
\text { (vol\%) }\end{array}$ & $\mathrm{E}_{\mathrm{Fe}}(\%)$ & $\mathrm{E}_{\mathrm{Sc}}(\%)$ & $\beta_{\mathrm{Fe} / \mathrm{sc}}$ \\
\hline 1 & 5 & 71.61 & 0.88 & 284.1 \\
\hline 2 & 10 & 80.65 & 1.26 & 326.6 \\
\hline 3 & 20 & 85.81 & 1.34 & 445.2 \\
\hline 4 & 30 & 88.71 & 1.72 & 449.0 \\
\hline 5 & 40 & 87.74 & 1.59 & 442.9 \\
\hline
\end{tabular}

\subsubsection{The effect of modifier concentration}

Although $\mathrm{N}_{235}$ has a good property on separation of $\mathrm{Sc}^{3+}$ and $\mathrm{Fe}^{3+}$, the separation rate of organic phase and aqueous phase is slow and easy to appear a third phase in organic phase. In order to solve this problem, a modifier Sec-Octyl alcohol was added into the organic phase. In this experiments, the concentration of Sec-Octyl alcohol was varied from $0 \mathrm{vol} \%$ to $25 \mathrm{vol} \%$ with other parameters constant.

Table 2. Effect of modifier concentration on extraction.

\begin{tabular}{|c|c|c|c|c|c|}
\hline No. & $\begin{array}{c}\text { Sec-Octyl alcohol } \\
\text { concentration } \\
\text { (vol\%) }\end{array}$ & $E_{\mathrm{Fe}}(\%)$ & $\mathrm{E}_{\mathrm{Sc}}(\%)$ & $\beta_{\mathrm{Sc} / \mathrm{Fe}}$ & $\begin{array}{l}\text { The third } \\
\text { phase }\end{array}$ \\
\hline 1 & 0 & 85.65 & 1.34 & 445.2 & existent \\
\hline 2 & 5 & 85.32 & 1.34 & 427.9 & non-existent \\
\hline 3 & 10 & 85.16 & 1.30 & 435.7 & non-existent \\
\hline 4 & 15 & 85.16 & 1.26 & 449.7 & non-existent \\
\hline 5 & 20 & 80.00 & 1.28 & 308.5 & non-existent \\
\hline 6 & 25 & 71.61 & 1.20 & 207.7 & non-existent \\
\hline
\end{tabular}

No difficulty was encountered in phase separation when adding Sec-Octyl alcohol. As shown in Table 2, the change of $\mathrm{Fe}^{3+}$ extraction rate is small with Sec-Octyl alcohol less than $15 \%$, but sharply dropped when it exceeded $15 \%$, while the change of $\mathrm{Sc}^{3+}$ is almost negligible. This may be due to the formation of complexation between Sec-Octyl alcohol and $\mathrm{N}_{235}$ thus reduce the effective concentration of extractant then prevented the combination of $\mathrm{Fe}^{3+}$ and $\mathrm{N}_{235}$. Therefore, the concentration of Sec-Octyl alcohol should be no more than $15 \mathrm{vol} \%$.

\subsection{Solvent extraction of scandium}

EHPAEH is a kind of organophosphorus acids extractant which is similar to DE2HPA. But EHPAEH is easier to be stripped owing to its weaker extraction ability. In addition, it is more suitable for extracting $\mathrm{Sc}^{3+}$ from high acidity solution[4]. In this experiment, 
EHPAEH is employed to extract $\mathrm{Sc}^{3+}$. The extraction principles are as follows[4]:

(1) in low acidity system:

(2) in high acidity system:

$$
\mathrm{Sc}^{3+}+3 \mathrm{HA} \rightarrow \mathrm{ScA}_{3}+3 \mathrm{H}^{+}
$$

$$
\mathrm{Sc}^{3+}+\mathrm{nHA} \rightarrow \mathrm{Sc}(\mathrm{HA})_{\mathrm{n}}{ }^{3+}
$$

It can be seen from reaction Eq. (1) and (2) that cation exchange reaction exits in low acidity solution and solvation exits in high acidity solution[4,12].

The effects of experimental parameters, such as aqueous $\mathrm{pH}$, EHPAEH concentration, and phase volume ratio are investigated to determine the optimum operating conditions.

Poor phase separation easily appears in the extraction process when organic phase contained only EHPAEH in sulfonated kerosene. After adding $15 \mathrm{vol} \%$ sec-Octyl alcohol as modifier, phase separation improvs significantly.

\subsubsection{Effect of $\mathrm{pH}$}

In the experiment, $\mathrm{pH}$ was regulated in the range of $0 \sim 2.0$ (under the following conditions) with 5 vol\% EHPAEH in sulfonated kerosene, phase volume ratio $\mathrm{O} / \mathrm{A}$ ratio of $1: 1$ and the temperature of $25^{\circ} \mathrm{C}$ ).

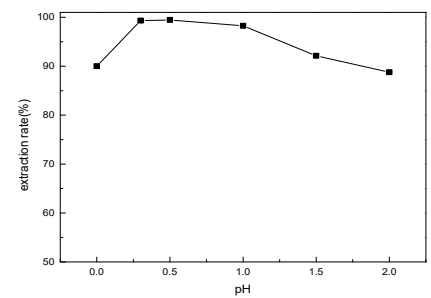

Fig. 1. Effect of solution $\mathrm{pH}$ on extraction rate.

It can be seen from Fig. 1 that at beginning, with the increase of $\mathrm{pH}$, the extraction rate of $\mathrm{Sc}^{3+}$ gradually increased then significantly decreased after $\mathrm{pH}$ greater than 0.9 . The preferable $\mathrm{pH}$ range for scandium extraction is $0.3 \sim 1.0$. The extraction rate gradually decreased with $\mathrm{pH}$ greater than 1.0, possibly caused by impurity irons hydrolysis. The initial $\mathrm{pH} 0.3$ is in the appropriate range, so directly extraction without adjusting $\mathrm{pH}$ is feasible.

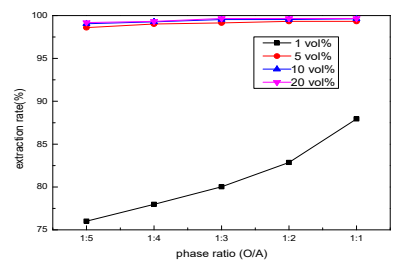

Fig. 2. Effect of phase ratio on extraction rate with different EHPAEH concentration.

\subsubsection{Effect of EHPAEH concentration and phase volume ratio}

Fig. 2 shows the effect of phase volume ratio $\mathrm{O} / \mathrm{A}$ on the $\mathrm{Sc}^{3+}$ extraction rate at different EHPAEH concentrations. Extraction rate increased with both increasing concentrations and increasing volume phase ratio. But lower $\mathrm{O} / \mathrm{A}$ is beneficial to concentrating scandium in the organic phase[4]. Scandium has more charge and smaller ionic radius which shows the great electron affinity and extractability, therefore, the selectivity of scandium over other metals is increased with low EHPAEH concentration. Since extraction rate doesn't increase obviously with the concentration of EHPAEH over $5 \mathrm{vol} \%$ and O/A over $1: 5,5 \mathrm{vol} \%$ and $1: 5$ is the decent concentration and phase volume ratio, respectively. 


\subsection{Stripping of scandium}

\subsubsection{Effect of hydrochloric acid concentration and phase volume ratio}

Lanthanide element has the characteristic of lanthanide contraction. It means the atomic radius of the sixth periodic element is smaller than the fifth. From lanthanum to lutetium with the increase of nuclear charge, the effective nuclear charge gradually increases, which cause the atomic volume reduced. Consequently the heavy rare earth elements with larger atomic number has more smaller radius. Scandium that located in the forth periodic is similar to heavy rare earth elements in terms of atomic radius. Therefore it requires high hydrochloric acid concentration in the stripping process[4].

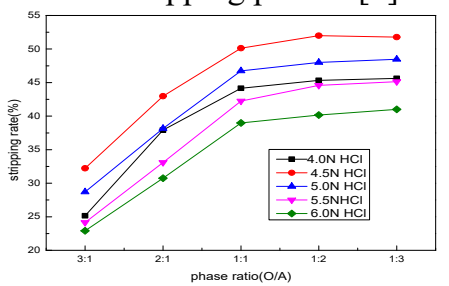

Fig. 3. Effect of phase ratio on stripping rate with different concentration of hydrochloric acid.

The effect of phase volume ratio $\mathrm{O} / \mathrm{A}$ with different hydrochloric acid concentration on stripping rate of scandium was investigated. Fig. 3 shows that at the outset the stripping rate of scandium gradually increased with the increasing acid concentration and it reaches the maximum of $50.13 \%$ with $4.5 \mathrm{~mol} / \mathrm{L} \mathrm{HCl}$ then begin to drop. Similarly, with the increase of phase volume ratio(O/A), the stripping rate of scandium gradually increased, then stables in a certain range with phase volume ratio over 1:1.

\subsubsection{Effect of temperature}

Scandium was stripped from scandium-loaded organic phase at different temperatures with the fixed condition of $4.5 \mathrm{~N} \mathrm{HCl}$, phase ratio $\mathrm{O} / \mathrm{A}$ of $1: 1$. Fig. 4 shows that at beginning with the increase of temperature, the stripping rate of $\mathrm{Sc}^{3+}$ gradually increased, but it only increased by $0.91 \%$ (from $80.36 \%$ to $81.27 \%$ ) when temperature increased from $65^{\circ} \mathrm{C}$ to $85^{\circ} \mathrm{C}$. Furthermore, high temperature easily led to the organics. To sum up, $65^{\circ} \mathrm{C}$ is appropriate.

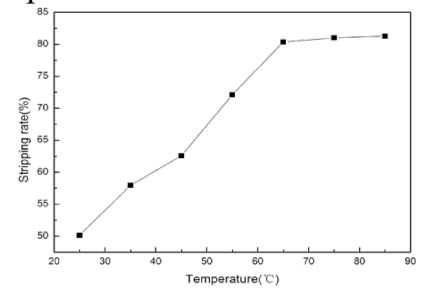

Fig. 4. Effect of temperature on stripping rate. rate.

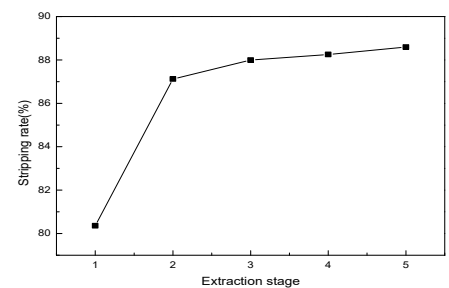

Fig. 5. Effect of stripping stage on stripping

\subsubsection{Effect of stripping stage}

According to Fig.5, the stripping rate of scandium is improved by increasing stripping stage. After two stages, the stripping rate reached $87.12 \%$ then increased slowly. After certain treatment, the organic solvent is reused for extraction. 


\section{Conclusion}

$\mathrm{N}_{235}$ demonstrates better selectivity for $\mathrm{Fe}^{3+}$ and $\mathrm{Sc}^{3+}$. More than $99 \% \mathrm{Fe}^{3+}$ in synthetic solution is extracted into organic phase while little scandium is co-extracted. The separation factor $\beta_{\mathrm{Fe} / \mathrm{sc}}$ reaches 5416.4 with the organic system consisting of $20 \mathrm{vol} \% \mathrm{~N}_{235}+15 \mathrm{vol} \%$ Sec-Octyl alcohol +65 vol\% sulfonated kerosene, phase volume ratio O/A of $1: 1$, temperature of $25^{\circ} \mathrm{C}$, and extraction stages of three.

EHPAEH has a high scandium extraction ability, even small amount can acquire good results. The extraction rate of $\mathrm{Sc}^{3+}$ reaches $98.59 \%$ with the organic system consisting of 5 vol\% EHPAEH, 15 vol\% sec-Octyl alcohol +80 vol\% sulfonated kerosene, phase volume ratio $(\mathrm{O} / \mathrm{A})$ of $1: 5$, at $\mathrm{pH} 0.3$.

Finally the Sc extracted complexes is stripped by hydrochloric acid. With increasing of temperature and hydrochloric acid concentration, the stripping rate of $\mathrm{Sc}^{3+}$ increases quickly but tend to be smooth finally. Under the conditions of hydrochloric acid concentration $4.5 \mathrm{~N}$, temperature of $65^{\circ} \mathrm{C}$, stripping stages of two, $87.12 \%$ scandium in organic phase returns into aqueous phase.

\section{References}

1. Wang W W, Pranolo Y and Cheng C Y. Metallurgical processes for scandium recovery from various resources: A review[J]. Hydrometallurgy, 2011, 108: 100-108.

2. $\mathrm{Xu} \mathrm{S} \mathrm{Q} \mathrm{and} \mathrm{Li} \mathrm{S} \mathrm{Q}$. Review of the extractive metallurgy of scandium in China (1978-1991)[J]. Hydrometallurgy, 1996, 42: 337-343.

3. Zhang Z B and Zhang Z H. Scandium resources and its extraction technology[J]. Yunnan Metallurgy, 2006, 5: 23-25.

4. Rongjun M. Principles on hydrometallurgy[M]. Beijing: Metallurgical Industry Press, 2007.

5. Ditze A and Kongolo K. Recovery of scandium from magnesium, aluminium and iron scrap[J]. Hydrometallurgy, 1997, 44: 179-184.

6. Wu D B, Niu C J, Li D Q and Bai Y. Solvent extraction of scandium(III), yttrium(III), lanthanum(III) and gadolinium(III) using Cyanex 302 in heptane from hydrochloric acid solutions[J]. Journal of Alloys and Compounds, 2004, 374: 442-446.

7. Zhang $\mathrm{P}$ W, You S T, Zhang L, Feng S and Hou S H. A solvent extraction process for the preparation of ultrahigh purity scandium oxide[J]. Hydrometallurgy, 1997, 47: 47-56.

8. Kostikova G V, Danilov N A, KrylovG Y S. Extractin of Scandium from Various Media with Triisoamyl Phosphate: 3. Development of Extractive Refining of Scandium[J], Radiochemistry, 2006, 48(5).

9. Liu Z B and Li H X. Metallurgical process for valuable elements recovery from red mud-A review[J]. Hydrometallurgy, 2015, 155:29-43.

10. Srnirnov D I and Molchanova T V. The investigation of sulphuric acid sorption recovery of scandium and uranium from the red mud of alumina production[J]. Hydrometallurgy, 1997, 45: 249-259.

11. Petropulu M O, Lyberopulu T and Parissakis G. Selective separation and determination of scandium from yttrium and lanthanides in red mud by a combined ion exchange/solvent extraction method[J]. Analytica Chimica Acta, 1995, 315: 231-237.

12. Zhou H L, Li D Y, Tian Y J and Chen Y F. Extraction of scandium from red mud by modified activated carbon and kinetics study[J]. Rare Metals, 2008, 3: 223-227. 